\title{
Des gènes pour les dystrophies rétiniennes des enfants
}

Josseline Kaplan
Jean-Michel Rozet
Sylvie Gerber
Agnès Camuzat
Éric Souied
Dominique Bonneau
Dominique Larget-Piet
Hélène Dollfus
Jean-Louis Dufier
Marie-Louise Briard
Jean Frézal
Arnold Munnich

Les dystrophies rétiniennes héréditaires constituent une cause fréquente de malvoyance grave de l'enfant. Elles représentent un groupe hétérogène au plan clinique, génétique et moléculaire au sein duquel ont pu être identifiées trois formes particulièrement sévères : la maladie de Usher de type I, la maladie de Stargardt et l'amaurose congénitale de Leber qui comptent parmi les plus graves des déficits sensoriels de l'enfant. Le premier gène responsable du syndrome de Usher I a été localisé sur le chromosome $14 q 32$ dans un isolat géographique français (Poitou). Puis, grâce à la carte génétique du Généthon, le gène de la maladie de Stargardt a été localisé sur le bras court du chromosome lp21-p13. Enfin, grâce à l'élégante méthode de l'homozygotie par filiation dans les familles consanguines, le premier gène responsable de l'amaurose congénitale de Leber a été repéré sur l'extrémité terminale du bras court du chromosome 17. L'identification de ces trois gènes permettra de contribuer à une meilleure connaissance de la physiopathologie des dystrophies rétiniennes humaines par l'analyse des protéines incriminées dans les processus dégénératifs de la rétine avec l'espoir de voir s'engager des recherches à visée thérapeutique pour ces affections qui, à l'heure actuelle, évoluent toutes inexorablement vers la cécité.

\footnotetext{
ADRESSE

J. Kaplan : médecin généticien, chargée de recherche $\dot{a}$ l'Inserm. J.M. Rozet : ingénieur d'études. S. Gerber : ingénieur d'études. A Camuzat : ingénieur d'études. E. Souied : ophtalmologiste, interne des hopitaux de Paris. D. Bonneau : médecin généticien, praticien hospitalier. D. Larget-Piet : interne en pharmacie. H. Dollfus : ophtalmologiste, chef de clinique des hôpitaux de Paris. J.L. Dufier : professeur d'ophtalmologie, médecin des hôpitaux de Paris, chef du service d'ophtalmologie de l'hôpital Necker-Enfants-Malades. M.L. Briard : médecin généticien, directeur de recherche à l'Inserm. J. Frézal : ancien professeur de génétique médicale, médecin des hôpitaux de Paris. A. Munnich : professeur de génétique médicale, médecin des hopitaux de Paris, directeur de l'U. 393 de l'Inserm, chef du service de génétique médicale de l'hôpital NeckerEnfants-Malades. Unité de recherches sur les handicaps génétiques de l'enfant, Inserm U. 393, centre de génétique médicale, hôpital des Enfants-Malades, 149, rue de Sèvres, 75743 Paris Cedex 15, France.
}

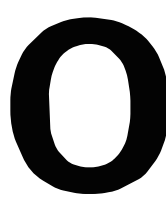
n désigne sous le terme de dystrophies rétiniennes des affections dégénératives atteignant de façon diffuse ou localisée les cellules photoréceptrices de la rétine que sont les cônes et les bâtonnets. Ces cellules participent à la couche la plus interne de la rétine et convertissent l'énergie électromagnétique du photon lumineux en un influx nerveux qui sera transmis au cerveau par l'intermédiaire du nerf optique [1]. Bien que certaines de ces affections soient des maladies de l'adulte, elles représentent une des grandes causes d'amblyopies et céci- tés de l'enfance, touchant environ $30 \%$ des enfants scolarisés dans les instituts pour déficients visuels [2]. La prévalence de ces affections, toutes formes confondues, a été estimée à 1/3000 habitants [3-6]. L'incidence des nouveaux cas reconnus chaque année est de 6 par million d'habitants, ce qui représente 330 nouveaux cas par an en France. On estime le nombre de patients atteints de dystrophie rétinienne à près de 25000 en France, 200000 en Europe et plus d'un million dans le monde. De nombreuses études ont souligné l'hétérogénéité des affections rétiniennes et la nécessité impérieuse de 


\section{RÉFÉRENCES}

1. Stryer $\mathbf{L}$. Cyclic GMP cascade of vision. Annu Rev Neurosci 1986 ; 9 : 87-119.

2. Feingold J, Briard ML, Kaplan J, Bonaiti C, Crouzet J, Demailly ML, Morel J, Delthil $\mathrm{S}$. Fréquence des affections héréditaires dans les instituts de déficients visuels. $J$ Genet Hum 1976 ; 24 : 85-91.

3. Ammann F, Klein D, Franceschetti A. Genetic and epidemiological investigation of pigmentary degeneration of the retina and allied disorders in Switzerland. $J$ Neurol S $i$ $1965 ; 2: 183-6$.

4. Boughman JA, Conneally PM, Nance WE. Population genetic studies of retinitis pigmentosa. Am J Hum Genet 1980 ; 32 : 223-34.

5. Bundey S, Crews J. A study of retinitis pigmentosa in the city of Birmingham. I. Prevalence. J Med Genet 1984 ; 21 :417-20.

6. Bunker $\mathrm{CH}$, Berson EL, Bromley WC, Hayes RP, Roderick TH. Prevalence of retinitis pigmentosa in Maine. Am J Ophthalmol $1984 ; 97: 357-65$.

7. Pagon RA. Retinitis pigmentosa. Surv Ophthalmol 1988 ; 33 : $137-77$.

8. Kaplan J, Bonneau D, Frézal J, Munnich A, Dufier JL. Clinical and genetic heterogeneity in retinitis pigmentosa. Hum Genet $1990 ; 85: 635-42$.

9. Merin S, Auerbach E. Retinitis pigmentosa. Surv Ophthalmol 1976 ; 20 : 303-46.

10. Fishman GA Retinitis pigmentosa : visual loss. Arch Ophthalmol 1978; 96 : 1185-8.

11. Jay M. Figures and fantasies : the frequencies of the different genetic forms of retinitis pigmentosa. Birth Defects $1982 ; 18$ : 167-73.

12. Boughman JA, Fishman GA. A genetic study of retinitis pigmentosa. $\mathrm{Br} J$ Ophthalmol $1983 ; 67: 449-54$.

13. Bundey S, Crews J. A study of retinitis pigmentosa in the city of Birmingham. II. Clinical and genetic heterogeneity. $J \mathrm{Med}$ porter un diagnostic exact chez les patients atteints [7]

En 1988, grâce à la mise en place d'une consultation spécialisée multidisciplinaire à l'Hôpital Laënnec $(\mathrm{Pa}-$ ris), nous avons entrepris une classification prospective des dystrophies rétiniennes. Les patients reçus à cette consultation bénéficient d'une exploration ophtalmologique clinique et paraclinique approfondie et d'une consultation de génétique avec établissement de l'arbre généalogique, relevé de l'origine géographique des familles et recherche d'une éventuelle consanguinité. Un premier bilan, après 18 mois de fonctionnement de cette consultation, avait permis d'établir, en juin 1989, les étiologies des dystrophies rétiniennes non syndromiques dans un échantillon de 150 patients [8] (Tableau I).

\section{Les rétinopathies pigmentaires}

Les rétinopathies pigmentaires isolées représentent le chef de file des dystrophies rétiniennes héréditaires. Elles résultent de la dégénérescence du couple "épithélium-pigmentairephotorécepteur" et constituent un groupe extraordinairement hétérogène de maladies, non seulement au plan clinique mais aussi au plan génétique et moléculaire. Au plan clinique, la forme la plus habituelle se manifeste par une atteinte primaire des bâtonnets. Ces photorécepteurs, disséminés sur l'ensemble de la rétine, ont pour fonction la vision en basse luminance et l'obtention d'un large champ visuel. Aussi n'est-il pas surprenant que les premières manifestations de ces rétinopathies pigmentaires soient une gêne à la vision crépusculaire et une réduction concentrique du champ visuel. Après plusieurs années d'une évolution dont la durée varie selon le type clinique, s'installe progressivement une atteinte des cônes localisés au pôle postérieur de la rétine (macula) qui ont pour fonction la vision diurne, la tolérance à la lumière et la discrimination colorée. C'est ainsi que cette atteinte secondaire des cônes se traduira par une baisse souvent rapide de l'acuité visuelle, une intolérance à la lumière solaire et un trouble de la vision des couleurs. L'évolution naturelle des rétinopathies pigmentaires, en l'absence de tout traitement spécifique conduit inéluctablement vers la cécité.

Il existe des "formes inverses de rétinopathies pigmentaires isolées " avec atteinte primaire des cônes et atteinte secondaire des bâtonnets. Ces formes peuvent être difficiles à distinguer des dystrophies rétiniennes purement maculaires. La terminologie anglo-saxonne est plus adaptée à la désignation de ces deux grands types de rétinopathies pigmentaires puisque les formes traditionnelles sont appelées rod-cone dystrophies alors que les formes inverses sont nommées cone rod dystrophies. Cette désignation est en passe d'envahir le langage médical en France.

Tous les modes de transmission ont été décrits depuis fort longtemps pour ces affections [9-14]. Dans l'étude que nous avons menée, nous avons retrouvé cet éventail des différents modes héréditaires [8] (Tableau II)

Les formes dominantes autosomiques ont été classées depuis les travaux de Massof et Finkelstein [15] en deux sous-groupes : le type I (ou forme diffuse), le plus rare, et le type II (ou forme régionale), le plus fréquent. Le type I est caractérisé par une perte généralisée de la fonction des bâtonnets sur l'ensemble de la rétine avec une relative préservation des cônes au début. L'atteinte commence dans la première décennie de la vie et entraîne un handicap visuel grave dès l'âge de 30 ans. Le type II comporte un dysfonctionnement des bâtonnets dans certaines régions de la rétine avec un déficit du champ visuel correspondant, alors que d'autres régions sont intactes. Cette forme commence à la fin de la seconde décennie, évolue très lentement et ne grève la fonction visuelle centrale que vers l'âge de 50 ans ou au-delà. Pour déterminer la place qu'occupent les mutations de novo dans ces formes dominantes, nous avons étudié le rôle de l'âge paternel et avons ainsi pu montrer, dans un petit nombre de familles, un âge paternel élevé à l'origine de ces néomutations $(38,8 \pm 4,7$ ans, $p<0,001)$ [8]. Ces constatations prennent toute leur valeur lorsqu'on les compare à celles retrouvées dans les cas sporadiques (voir plus loin). 


\begin{tabular}{|l|}
\hline \multicolumn{2}{|c|}{ Tableau I } \\
\multicolumn{2}{|c|}{ ÉTIOLOGIES DES DYSTROPHIES } \\
RÉTINIENNES \\
NON SYNDROMIQUES \\
\hline $\begin{array}{l}|c| \\
\text { - Rétinopathie pigmentaire } \\
\text { isolée }\end{array}$ \\
- Syndrome de Usher \\
- Maladie de Stargardt et \\
$\begin{array}{l}\text { fundus flavimaculatus } \\
\text { - Amaurose congénitale } \\
\text { de Leber }\end{array}$ \\
$\begin{array}{l}\text { - Dystrophie des cônes } \\
\text { - Choroïdérémie } \\
\text { - Cécité nocturne } \\
\text { congénitale stationnaire }\end{array}$ \\
\hline
\end{tabular}

\begin{tabular}{|l|}
\hline \multicolumn{2}{|c|}{ Tableau II } \\
DIFFÉRENTS MODES \\
HÉRÉDITAIRES DANS LES \\
RÉTINOPATHIES PIGMENTAIRES \\
\hline - Formes dominantes \\
$\begin{array}{l}\text { autosomiques } \\
\text { - Formes récessives } \\
\text { autosomiques } \\
\text { - Formes récessives } \\
\text { liées à I'X } \\
\text { - Fratries de garçons } \\
\text { - Cas sporadiques }\end{array}$ \\
\hline
\end{tabular}

Les formes récessives autosomiques ont été beaucoup moins étudiées au plan clinique. Elles étaient autrefois considérées comme les plus fréquentes et les plus sévères, mais nous n'avons pas été en mesure de le confirmer puisque la fréquence des rétinopathies pigmentaires récessives autosomiques est, dans les pays occidentaux, égale à celle des formes dominantes ; on retrouve au moins quatre sous-groupes cliniques : rodcone dystrophies précoces et sévères, rod-cone dystrophies tardives et modérées (phénotype identique au type II des rétinopathies pigmentaires isolées dominantes), rod-cone dystrophies liées à l'âge (débutant après l'âge de 60 ans) et cone-rod dystrophies. La consanguinité dans ce groupe (35\% des cas) est 50 fois plus élevée que dans la population française. La consanguinité élevée dans le groupe suggère une grande multiplicité des gènes en cause dans les formes récessives autosomiques, chacun de ces gènes ayant vraisemblablement une fréquence faible [8].

$m / s n^{\circ} 3$, vol. 11, mars 95
Les formes récessives liées au chromosome $\mathbf{X}$ ont toujours été considérées comme très sévères. Elles touchent les garçons dès la première ou la seconde décennie et évoluent rapidement vers la cécité à l'âge de 30-35 ans [16]. Grâce à l'analyse attentive du mode d'installation de la maladie, nous avons proposé d'individualiser deux formes de rétinopathies pigmentaires liées à l'X $\left(\mathrm{m} / \mathrm{s} n^{\circ} 8\right.$ vol. 8 , p. 876) : l'une, débutant très tôt, vers l'âge de 3-4 ans, par une baisse sévère de l'acuité visuelle, se traduisant par une forte myopie alors que le champ visuel est encore préservé, l'autre, débutant un peu plus tard, vers l'âge de 10 ans, par une gêne de la vision nocturne et une réduction du champ visuel [8]. Ces notions sont souvent enfouies dans la mémoire de ces patients interrogés à l'âge adulte et cela d'autant plus qu'à partir de l'âge de 15 ans environ, il existe un dysfonctionnement mixte des cônes et des bâtonnets dans les deux sousgroupes qui ne se distinguent donc que par leur mode et âge de début à une période où le diagnostic n'est pas évoqué puisque, dans le premier groupe, les enfants sont simplement considérés comme de grands myopes (voir plus loin). Les cas sporadiques sont les plus fréquents (43\% dans notre série), et ce taux élevé est retrouvé par tous les auteurs. Fait intéressant, nous avons retrouvé un taux de consanguinité de $10 \%$ dans l'ensemble des cas isolés (21\% pour les seules formes précoces et sévères), soit un taux de consanguinité 15 fois supérieur à celui de la population générale, suggérant donc que certains de ces cas isolés pourraient correspondre à des formes récessives autosomiques survenant isolément, ce qui n'a rien de surprenant compte tenu de la petite taille des familles humaines. La deuxième surprise vint $d u$ taux de masculinité. Compte tenu de la sévérité des formes récessives liées à l'X, nous avons séparé les formes les plus précoces et les plus graves de l'ensemble des cas sporadiques, et le sex-ratio dans cet échantillon fut estimé à 1,8, c'est-à-dire augmenté de façon très significative par rapport au sex-ratio des autres formes (1,05 pour les rétinopathies pigmentaires dominantes, 0,82 pour les rétinopathies pigmentaires récessives autosomiques, 0,95 pour l'ensemble des cas sporadiques). Ce très fort taux de masculinité suggère donc l'existence de mutations récentes sur le chromosome X dans le sous-groupe des cas isolés sévères [8]. Enfin, compte tenu de la fréquence du phénotype tardif et modéré des formes dominantes autosomiques, nous avons étudié l'âge paternel des malades atteints de ces formes modérées et avons retrouvé un âge moyen augmenté de façon significative $(33,8 \pm 9,8$ ans ; $p<$ $0,001)$ avec un pourcentage de pères de plus de 35 ans de $31,5 \%$ contre $16 \%$ dans la population générale. Cet âge paternel élevé suggère donc l'existence, au sein de cet échantillon, de mutations dominantes autosomiques survenant de novo [8].

\section{Corrélations \\ phénotype-génotype dans les rétinopathies pigmentaires liées au chromosome $X$ (RPLX)}

La première liaison génétique dans une rétinopathie pigmentaire a été établie, en 1984, par une équipe anglaise [17] sur le bras court du chromosome X (Xp11). Cette liaison étroite ne fut pas retrouvée par d'autres auteurs et le nombre de recombinaisons suggérait une grande distance génétique dans certaines familles [18]. Au demeurant, trois patients présentaient des délétions comprises entre Xp21.3 et Xp21.1 $[19,20]$. Dans ces cas, d'autres maladies liées à l'X étaient associées à la rétinopathie pigmentaire : maladie de Duchenne, granulomatose chronique et perte de l'antigène érythrocytaire KX (phénotype McLeod). Cela permit de suspecter la localisation en Xp21.1 d'un second gène de rétinopathie pigmentaire $[20,21]$. Ainsi, il existe pour les rétinopathies pigmentaires récessives liées à l'X un gène $R P 2$ situé en $\mathrm{Xp} 11.21-\mathrm{Xp} 11.41$ et un gène $R P 3$ situé en $\mathrm{Xp} 21.1$. Or, nous avions distingué deux formes différant par leur mode et âge de début [8]. Dans le but de contribuer à l'établissement de corrélations phénotype-génotype, nous avons entrepris une étude de liaison génétique dans neuf grandes familles de rétinopathies pigmentaires liées à l'X, quatre d'entre elles correspondant au premier phénotype et les cinq autres au second phénotype. Nous 


\section{RÉFÉRENCES}

14. McKusick VA. Mendelian inheritance in man, 8th ed. Baltimore: The Johns Hopkins University Press, 1989 : 804.

15. Massof RW, Finkelstein D. Two forms of autosomal dominant primary retinitis pigmentosa. Doc Ophthalmol 1981 ; 51 : 289-346.

16. Heckenlively IR, Yoser SL, Friedman LH, Oversier JJ: Clinical findings and common symptoms in retinitis pigmentosa. $A m J$ Ophthalmol $1988 ; 105$ : 504-14.

17. Bhattacharya SS, Wright AF, Clayton JF, Price WH, Phillips CI, McKeown CME, Jay M, Bird AC, Pearson PL, Southern EM, Evans HJ. Close linkage between X-linked retinitis pigmentosa and a restriction fragment length polymorphism identified by recombinant DNA probe L1.28. Nature 1984 ; 309: 253-5.

18. Nussbaum RL, Lewis RA, Lesko JG, Ferrel R. Mapping ophthalmological diseases. II. Linkage relationship of X-linked retinitis pigmentosa to $\mathrm{X}$ chromosome short arm markers. Hum Genet 1985 ; 70 : 45-50.

19. Francke U, Ochs HD, de Martinville B. Minor Xp21 chromosome deletion in a male associated with expression of Duchenne muscular dystrophy, chronic granulomatous disease, retinitis pigmentosa and Mc Leod syndrome. Am J Hum Genet 1985 ; 37 250-67.

20. de Saint-Basile G, Bohler MC, Fischer A Xp21 DNA microdeletion in a patient with chronic granulomatous disease, retinitis pigmentosa, and the Mc Leod phenotype. Hum Genet 1988 ; 80 : 85-9.

21. Ott J, Battacharya SS, Chen JD, Denton NMJ, Donald DJ, Dubay L. Localizing multiple $\mathbf{X}$ chromosome-linked retinitis pigmentosa loci using multilocus homogeneity tests. Proc Natl Acad Si USA 1990;87:7014 .

22. Kaplan J, Pelet A, Martin C, Delrieu O, Aymé S, Bonneau D, Briard ML, Hanauer A, Larget-Piet L, Lefrançois P, Michel-Awad A, Plauchu H, Dufier JL, Frezal J, Munnich A. Phenotype-genotype correlations in $\mathrm{X}$ linked retinitis pigmentosa. I Med Genet $1992 ; 29: 615-23$.

23. Jacobson SG, Roman AJ, Robey MG Iwata $\mathrm{T}$, Inana $\mathrm{G}$. X-linked retinitis pigmentosa : functional phenotype of an RP2. In vest Ophthalmol Vis Sci 1992 ; 33 : 3524.

24. Kaplan J, Guasconi G, Dufier JL, MichelAwad A, David A, Munnich A, Frezal J. Exclusion of linkage between D3S47 (C17) and ADRPII in two large families of moderate autosomal dominant retinitis pigmentosa : evidence for genetic heterogeneity.

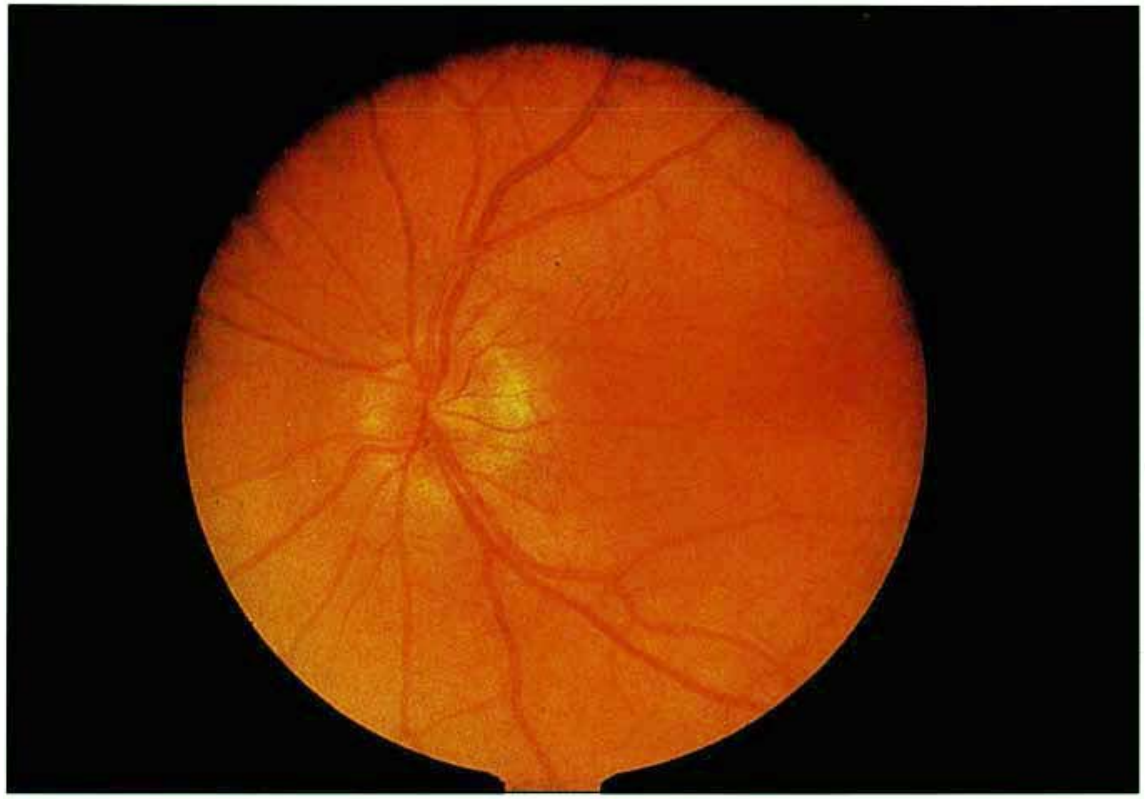

Figure 1. Fond d'œil normal.

avons utilisé des marqueurs polymorphes aux loci: DXS84 et OTC localisés en Xp2.1 définissant le locus RP3 et DXS7, DXS14 et DXS255 localisés en Xp1.1 définissant le locus RP2. Fait intéressant, dans les quatre familles de rétinopathies pigmentaires présentant une myopie très précoce (RPLX-A), le lod-score maximal fut obtenu au locus DXS255 (Z = 3,13 à $\theta=0$ ) alors que des valeurs négatives furent obtenues aux loci DXS84 et OTC. En revanche, dans les cinq familles pour lesquelles l'affection commence plus tardivement par une hespéralopie*, le lod-score maximal fut obtenu avec OTC $(\mathrm{Z}=4,16$ à $\theta=0)$ tandis que des valeurs d'exclusion furent obtenues aux loci DXS255 et DXS14. L'analyse multipoint montra que, pour le groupe RPLX-A, la position la plus probable du gène se situait au niveau du locus DXS255 correspondant au gène RP2 tandis que, pour le groupe RPLX-B, la position la plus probable se situait au niveau du locus OTC correspondant au gène $R P 3$ [22]. Cette étude souligne la valeur de l'interrogatoire soigneux des patients et de leur famille à la recherche des toutes premières manifestations de la maladie, car détecter des différences phénotypiques à un stade avancé peut être beaucoup plus difficile. Pour les

* Hespéralopie: cécité nocturne. rétinopathies pigmentaires liées à l'X, en effet, tous les patients présentent, à partir de l'âge de 15 ans environ, le même tableau clinique et la même évolution inexorable vers la cécité. Les travaux de Jacobson et al. [23] confirmèrent ces résultats en montrant, par l'étude du champ visuel et de l'électrorétinographie, pratiqués à un stade très précoce puis à des étapes ultérieures de l'évolution de la maladie, que le génotype $R P 2$ correspondait à un dysfonctionnement primaire des cônes, les bâtonnets étant altérés secondairement (cone-rod dystrophy), alors que le génotype $R P 3$ correspondait à un dysfonctionnement primaire des bâtonnets, les cônes étant altérés secondairement (rod-cone dystrophy). Cette corrélation clinico-génétique n'a pas qu'une valeur théorique; elle est de la plus grande importance dans les études génétiques réalisées pour répondre à l'interrogation des familles (établissement de statut des femmes apparentées aux patients et, a fortiori, diagnostic anténatal de la maladie).

\section{Les rétinites pigmentaires dominantes autosomiques (RPDA)}

En 1989, l'équipe de P. Humphries établit la première localisation d'un gène de RPDA [24]. En effet, après exclusion d'une grande partie du gé-

$$
m / s n^{\circ} 3, \text { vol. } 11 \text {, mars } 95
$$




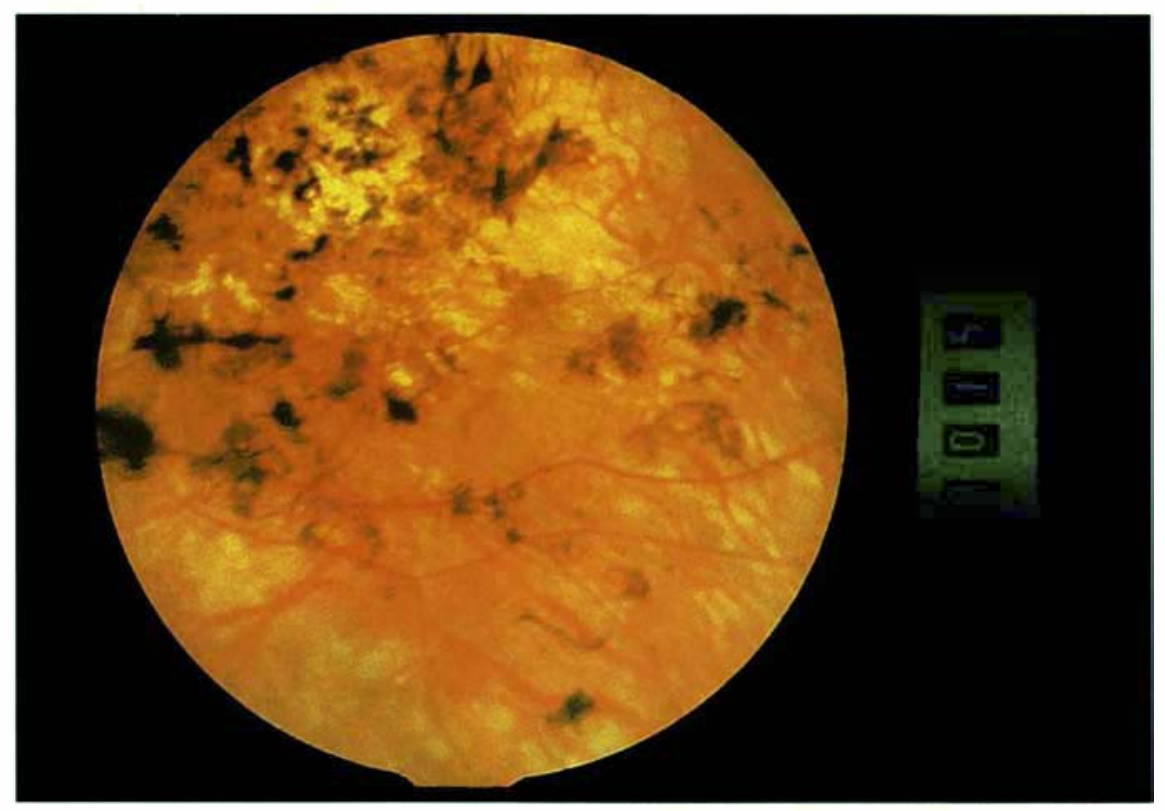

Figure 2. Fond d'œil dans un syndrome de Usher de type I. Notez les amas et les migrations pigmentaires disséminés sur la rétine.

nome, une coségrégation entre un marqueur polymorphe du chromosome 3 au locus D3S47 et le locus morbide fut trouvé dans une famille irlandaise [24]. Dans cette famille, qui comptait 50 individus atteints, s'observait une forme très précoce de la maladie, où la cécité nocturne apparaissait très jeune, l'évolution sévère amenant les sujets atteints à un handicap visuel important avant l'âge de 20 ans (RPDA type I). Nous avons voulu tester cette localisation dans les familles de rétinopathies pigmentaires dominantes autosomiques à début plus tardif et d'évolution moins sévère (type II). Pour ce faire, ont été réunies 20 familles de RPDA type II dont trois grandes familles sur quatre générations avec 24 individus atteints en vie et 32 indemnes. Ces travaux et ceux réalisés parallèlement par l'équipe de Humphries parvinrent à exclure le gène de type II du chromosome 3, mettant ainsi en évidence de façon claire l'hétérogénéité génétique des RPDA [25, 26].

Ce premier gène de RPDA sur le bras long du chromosome 3 (3q21-q22) présentait l'immense intérêt de se trouver dans une région contenant le gène de la rhodopsine humaine. Ce gène jouant un rôle majeur dans la cascade de la transduction visuelle se trouvait ainsi propulsé à la première place des gènes candidats pour les ré- s'agit toujours d'une affection récessive autosomique. Cette maladie est le plus souvent en cause chez les adultes sourds-aveugles. Elle représente environ $5 \%$ des surdités de l'enfant et $14 \%$ de l'ensemble des rétinopathies pigmentaires [8, 29].

Deux sous-groupes distincts ont été identifiés : le syndrome de Usher type I (USI) se définit par une surdité congénitale profonde (perte de plus de $100 \mathrm{db}$ ), interdisant l'acquisition du langage qui reste inintelligible même après de longues années de rééducation orthophonique. Les enfants atteints sont donc scolarisés dans des instituts pour malentendants. Ils apprennent la lecture labiale et le langage des signes. Le deuxième signe constant est un déficit vestibulaire bilatéral se traduisant dès les premiers mois de la vie par une hypotonie, un retard à l'acquisition de la marche (autour de 18 mois2 ans) et des troubles de l'équilibre pouvant aller jusqu'à une véritable ataxie. Enfin, à la fin de la première décennie, apparaissent les premiers signes d'une rétinopathie pigmentaire de type rod-cone dystrophy, qui va évoluer rapidement et grever la fonction visuelle dès l'âge de 20-25 ans, faisant de ces enfants sourds profonds, des adultes sourds-aveugles qui, par la perte de leur faculté de lecture labiale et du langage des signes, vont s'enfermer progressivement dans un isolement total. Le syndrome de Usher type II (USII) est une forme moins grave, avec une surdité moins sévère, souvent appareillable, permettant l'acquisition du langage, l'absence de déficit vestibulaire et une rétinopathie pigmentaire apparaissant à la fin de la seconde décennie et évoluant très lentement sur plusieurs dizaines d'années.

En 1990, Kimberling et al. [30] ont localisé le gène responsable de la forme modérée (Usher type II) sur le bras long du chromosome 1, grâce à une liaison forte avec un marqueur polymorphe au locus D1S81. A cette époque, ayant rassemblé six familles à cas multiples atteintes de la forme grave (Usher type I), nous avons éliminé l'hypothèse de formes alléliques au même locus établissant ainsi l'hétérogénéité, non seulement génétique, mais aussi clinique du syndrome de Usher. Puis, nous avons entrepris de construire une carte 


\section{RÉFÉRENCES}

25. McWilliam P, Farrar GJ, Kennar P, Bradley DG, Humphries MM, Sharp EM, McConnel DJ, Lawler M, Sheils D, Ryan C, Stevens $K$, Daiger SD, Humphries $P$. Autosomal dominant retinitis pigmentosa (ADRP) : localization of an $A D R P$ gene to the long arm of chromosome 3 . Genomics $1989 ; 5: 619-22$.

26. Farrar GI, McWilliam P, Bradley DG Kenna P, Lawler M, Sharp EM, Humphries MM, Eiberg $\mathbf{H}$, Conneally $\mathbf{M}$, Trofatter JA, Humphries P. Autosomal dominant retinitis pigmentosa : linkage to rhodopsin and evidence for genetic heterogeneity. Genomics $1990 ; 8: 35-40$.

27. Al-Maghtheh M, Gregory C, Inglehearn C, Hardcastle A, Battacharya S. Rhodopsin mutations in autosomal dominant retinitis pigmentosa. Hum Mutation 1993 ; 2 : 249-55.

28. Souied E, Gerber S, Rozet JM, Bonneau D, Dufier JL, Philip N, Coscas G, Munnich A, Kaplan J. Five novel missense mutations of rhodopsin gene in autosomal dominant retinitis pigmentosa. Hum Mol Genet 1994 ; $3: 1433-4$

29. Boughman JA, Vernon M, Shave RKA. Usher syndrome : definition and estimate of prevalence from two high-risk populations. Chronic Dis 1983 ; 36 : 595-603.

30. Kimberling WJ, Weston MD, Moller C, Davenport SL.H, Shugart YY, Priluck IA, Martini A, Milani M, Smith RJ. Localization of Usher syndrome type II to chromosome lq. Genomics $1990 ; 7: 245-9$.

31. Kaplan J, Gerber S, Bonneau D, Rozet JM, Delrieu O, Briard ML, Dollfus H, Ghazi I, Dufier JL, Frezal J, Munnich A. A gene for Usher syndrome type I (USH1A) maps to chromosome 14q. Genomics $1992 ; 14: 979$ 87.

32. Kimberling WJ, Moller CG, Davenport S, Priluck IA, Beighton PH, Greenberg J, Reardon W, Weston MD, Kenyon JB, Grunkemeyer JA, Pieke Dahl S, Overbeck LD, Blackwood DJ, Brower AM, Hoover DM, Rowland P, Smith RJH. Linkage of Usher syndrome type I gene (USH1B) to the long arm of chromosome 11 . Genomics $1992 ; 14$ : 988-94.

33. Smith RJH, Lee EC, Kimberling WJ, Daiger SP, Pelias MZ, Keats JB, Jay M, Bird A, Reardon W, Guest M, Ayyagari R, Hejtmancik JF. Localization of two genes for Usher syndrome type I to chromosome 11. Geno- d'exclusion en étudiant, par la méthode des lod-scores, la ségrégation conjointe entre le gène morbide et des marqueurs d'ADN polymorphes répartis tout le long du génome. Une liaison entre le gène responsable de US1 et plusieurs marqueurs du bras long du chromosome 14 a été mise en évidence (Zmax au locus D14S13 = $3,20$ à $\theta=0)\left(m / s n^{\circ} 1\right.$, vol. $9, p$. 98) [31]. Puis, nous avons démontré l'existence d'une hétérogénéité génétique puisque la localisation sur le chromosome 14 ne rendait compte que de dix familles sur quinze $(Z=$ 4,13 à $\theta=0$, test de Morton et test Homog significatifs $\mathrm{p}<0,01)$. Fait intéressant, ces dix familles étaient originaires de la même région du Poitou, d'un périmètre géographique de quelques kilomètres autour de la ville de Bressuire (Deux-Sèvres). L'hypothèse la plus vraisemblable est donc celle d'un effet fondateur à l'origine de l'affection dans ces familles qui ne se connaissent pas de lien de parenté proche [31]. Grâce à la banque de microsatellites du Généthon et à l'extension de notre échantillon (22 familles dont 11 poitevines, 9 françaises d'autres origines, 1 originaire d'Afrique du Nord et la dernière turque), la localisation de ce gène au locus D14S13 a pu être établie.

Un peu plus tard, deux équipes américaines obtenaient des résultats très intéressants en étudiant d'autres familles de Usher I, d'origines géographiques différentes. D'abord Kimberling obtenait une liaison étroite entre le gène responsable de l'affection dans des familles nord-américaines non acadiennes et un marqueur anonyme D11S527 localisé sur le bras long du chromosome 11 [32]. Parallèlement, Smith et Keats mettaient en évidence une liaison forte entre le gène responsable de la maladie dans des familles acadiennes de Louisiane et un marqueur anonyme (D11S419) localisé sur le bras court du chromosome 11 [33]. Fait intéressant, parmi les familles françaises non liées au 14, neuf étaient compatibles avec une liaison au chromosome 11q13.5 (test Homog significatif, $p<0,001)$. S'agissant du bras court du chromosome 11 (région 11p15), deux familles non liées au 14q ni au 11q étaient compatibles avec une localisation dans cette région. Enfin, aucune des localisations présomptives ne rendait comp- te des familles turques ou nord-africaines analysées. Il semble donc bien qu'il existe au moins quatre gènes responsables du syndrome de Usher de type I! De plus, force est de constater qu'aucun test d'hétérogénéité n'est capable de reconnaître l'existence de quatre gènes au sein d'un échantillon de familles humaines atteintes de maladies récessives autosomiques. Cette observation ajoute un obstacle supplémentaire à la cartographie primaire des maladies humaines génétiquement hétérogènes.

\section{Cartographie génétique de la maladie de Stargardt}

Dans le cadre très hétérogène des dystrophies rétiniennes héréditaires, existe une entité particulière, de transmission récessive autosomique, décrite par Karl Stargardt en 1909 [34]. Il s'agit d'une dystrophie maculaire pure (région centrale de la rétine) caractérisée par la survenue brutale entre 7 et 12 ans d'une baisse importante de l'acuité visuelle, d'évolution rapide, transformant en quelques mois ces enfants en amblyopes profonds. C'est la cause la plus fréquente de dégénérescence maculaire de l'enfant : elle rend compte de $7 \%$ de l'ensemble des dystrophies rétiniennes. Les enfants atteints de cette affection ne peuvent poursuivre une scolarité normale puisque en deux ou trois ans, ils ont une acuité visuelle centrale inférieure à $1 / 10$, mais leur rétine périphérique reste intacte tout au long de leur vie, leur permettant une déambulation relativement aisée.

A côté de ces formes typiques de la maladie de Stargardt, Franceschetti en 1965 [35] décrivit une autre forme de dystrophie maculaire avec taches qu'il nomma fundus flavimaculatus avec un aspect ophtalmoscopique très similaire à l'affection décrite par Stargardt, mais en différant par un début plus tardif, après l'âge de 20 ans et variable d'une famille à l'autre, et même d'un individu à l'autre à l'intérieur d'une même famille. L'évolution de ces formes est également beaucoup plus lente et le pronostic final meilleur. Dans les années qui suivirent, la majorité des cas décrits par Franceschetti furent 

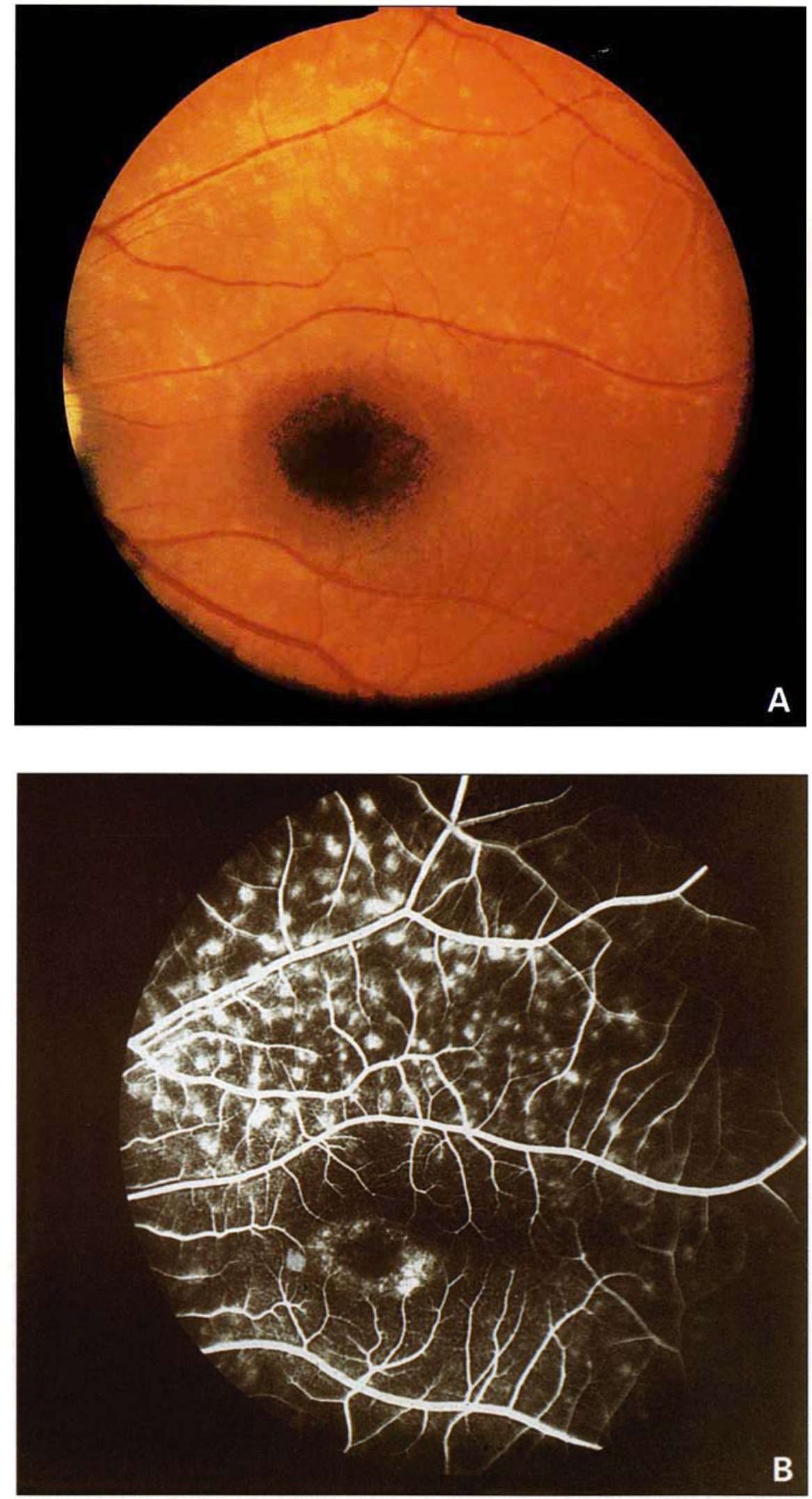

Figure 3. Fond d'œil (A), angiographie (B), d'une maladie de Stargardt. Notez l'atrophie maculaire (image en "œil de bœuf") et les tâches flavimaculées périmaculaires.

$\mathrm{m} / \mathrm{s} n^{\circ} 3$, vol. 11 , mars 95 considérés comme similaires à ceux décrits par Stargardt et les deux phénotypes furent considérés comme des manifestations différentes de la même maladie. Toutefois, aucune étude de cartographie n'avait été rapportée. En 1993, nous avons réuni huit grandes familles à cas multiples de la maladie de Stargardt comprenant 24 sujets atteints et 42 apparentés. Les critères d'inclusion dans cet échantillon furent très stricts et correspondaient stricto sensu à la description faite par Stargardt en 1909. L'allélisme de la maladie de Stargardt avec les céroïdes lipofuscinoses (CLN1, CLN3) fut d'abord testé car des inclusions de lipofuscine sont observées dans les cellules de l'épithélium pigmentaire dans la maladie de Stargardt. Par ailleurs, l'atteinte de la forme juvénile de céroïde lipofuscinose (CLN3) était évocatrice d'une dystrophie rétinienne centrale. Nous avons pu rejeter formellement l'allélisme en excluant la localisation du gène responsable de la maladie de Stargardt des deux régions explorées : 1 p32 pour la forme infantile de céroïde lipofuscinose (CLN1) et 16p12-p11 pour la forme juvénile [36]. Nous avons alors poursuivi la carte génétique d'exclusion du chromosome 1 en raison de la présence, sur ce chromosome, de la sous-unité $\alpha$ de la transducine spécifique des cônes [37]. Une très forte liaison au locus D12S236 put être mise en évidence $(\mathrm{Zmax}=6,88$ à $\theta=0,02)$. Sept autres marqueurs de la région testés dans nos huit familles donnèrent des lod-scores positifs sans aucun argument pour une hétérogénéité génétique et avec une localisation vraisemblable du gène au locus D1S435. Le gène responsable de la maladie de Stargardt était donc localisé sur le bras court du chromosome 1 (région 1p21-p13) et l'affection semblait génétiquement homogène $\left(m / s \quad n^{\circ} 12\right.$, vol. 9, p. 1418) [38].

Après ce travail sur la forme typique de maladie de Stargardt, nous avons étudié les formes dites Stargardt-like ou fundus flavimaculatus, à transmission récessive autosomique puisque de nombreux auteurs considéraient les deux phénotypes comme des manifestations différentes d'une même affection. Ainsi, dans une des dernières livraisons de la revue Archives of Ophthalmology, Richard Weleber dé- 


\section{RÉFÉRENCES}

34. Stargardt K. Uber familiäre, progressive degeneration under makulagegend des Auges. Albrecht von Graefes Arch Ophthalmol $1909 ; 71: 53450$.

35. Franceschetti A, François J. Fundus flavimaculatus. Arch Ophthalmol 1965 ; 25 : 505 30

36. Gerber S, Odent S, Postel-Vinay A, Janin N, Dufier JL, Munnich A, Frezal J, Kaplan J Stargardt's disease is not allelic to the genes for neuronal ceroid lipofuscinoses. $\int \mathrm{Med}$ Genet 1994 ; 31 : 222-3.

37. Wilkie TM, Gilbert DJ, Olsen AS, Chen XN, Amatruda TT, Korenbert JR, Trask BJ, De Jong P, Reed RR, Simon MI, Jenkins NÁ Copeland NG. Evolution of the mammalian $\mathrm{G}$ protein $\alpha$-subunit multigene family. Nature Genet $1992 ; 1: 85-91$.

38. Kaplan J, Gerber S, Larget-Piet D, Rozet JM, Dollfus H, Dufier JL, Odent S, Postel-Vinay A, Janin N, Briard ML, Frezal J, Munnich A. A gene for Stargardt's disease (fundus flavimaculatus) maps to the short arm of chromosome 1. Nature Genet 1993 ; 5 : 30811.

39. Weleber RG. Stargardt's macular dystrophy. Arch Ophthalmol 1994 ; 112 : 752-4.

40. Gerber S, Rozet JM, Bonneau D, Souied E, Camuzat A, Dufier JL, Amalric P, Weissenbach J, Munnich A, Kaplan J. A gene for late-onset fundus flavimaculatus with macular dystrophy maps to chromosome 1 p13. Am J Hum Genet 1995 (sous presse).

41. Leber T. Uber retinitis pigmentosa und angaborene amaurose. Graefes Arch Klin Exp Ophthalmol $1869 ; 15$ : 13-20.

42. Vogt O. Psychiatrisch wichtige tatsachen der zoologisoh botanischen systematik. $Z$ Neurol Psychiatrie 1926 ; 101 : 805-32.

43. Weil D, Mattei MG, Passage E, VanCong N, Pribula-Conway D, Mann K, Deutzmann R, Timpl R, Chu ML. Cloning and chromosomal localization of human genes encoding the three chains of type VI collagen. Am J Hum Genet 1988 ; 42 : 435-45.

44. Ueno S, Nakamura $Y$, Takahashi M, Tarui $S$, Sadaki H. "Non-prealbumin-related" familial amyloid polyneuropathy. Neurology

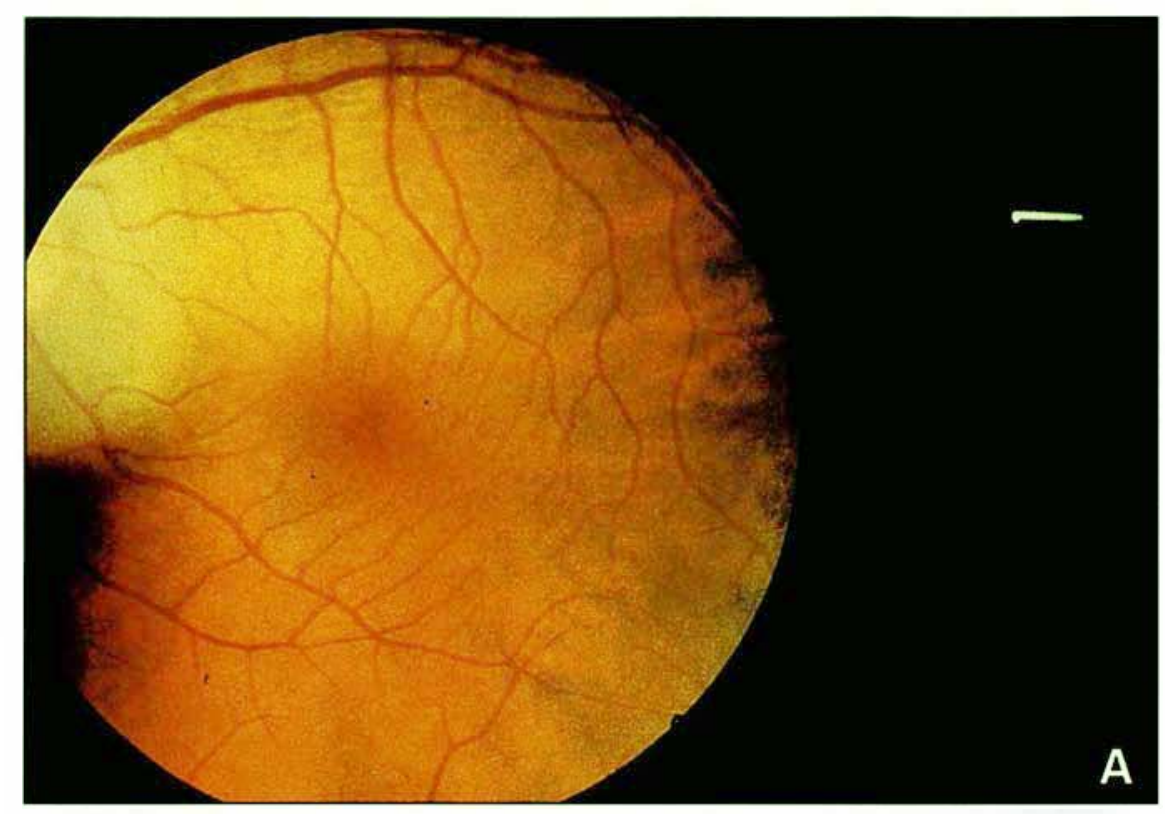

Figure 4. Fond d'œil dans la maladie de Leber. A. A la naissance. B. Après plusieurs années d'évolution. Notez que le fond d'œil est normal à la naissance. Après plusieurs années d'évolution, les artères rétiniennes sont très grêles et le nerf optique est atrophié.

fendait l'idée selon laquelle il fallait parler de maladie de Stargardt si la perte d'acuité visuelle et la dégénérescence maculaire commençaient dans la première décennie de la vie, alors que si la maladie commençait plus tard dans la vie, avec une évolution plus lente et une meilleure préservation de l'acuité visuelle, le terme fundus flavimaculatus était préférable [39].

Pour tester l'allélisme de ces formes tardives, quatre familles comprenant 11 sujets atteints et 12 apparentés ont été rassemblées et une étude de déséquilibre de liaison (linkage) utilisant les mêmes marqueurs que ceux ayant permis la localisation du gène de la maladie de Stargardt a été entreprise. Sans aucun événement de recombinaison, une forte liaison génétique avec les mêmes loci a été mise en évidence (lod-score maximal $=5,20$ ). Ces résultats suggèrent une homogénéité génétique de la maladie de Stargardt et d'autres dystrophies maculaires de début plus tardif et d'évolution plus lente. Il est important de noter que, parmi les quatre familles analysées, l'une comprenait trois sœurs ayant débuté leur maladie entre 60 et 65 ans [40].

\section{Cartographie génétique de l'amaurose congénitale de Leber}

En 1869, Theodor Leber décrivit pour la première fois sous ce nom d'amaurose congénitale une entité clinique considérée comme une rétinite pigmentaire in utero [41]. Il nota son caractère héréditaire et sa transmission récessive autosomique ainsi que la fréquence de la consanguinité dans les familles.

L'amaurose congénitale de Leber fut laissée pour compte pendant plusieurs années. Près de 85 ans après sa description, l'apparition de l'électrorétinographie (ERG) suscita un regain d'intérêt pour cette maladie lorsque fut mise en évidence une extinction totale de l'ERG dès les premiers mois de la vie (en l'absence d'altération du fond d'œil), témoignant d'une atteinte majeure des deux types de photorécepteurs : cônes et bâtonnets. L'amaurose congénitale de Leber est donc la dystrophie rétinienne la plus grave puisqu'elle est responsable de cécité néonatale. L'abolition de l'ERG dès les tout premiers mois de la vie est un élément clé pour porter le diagnostic 


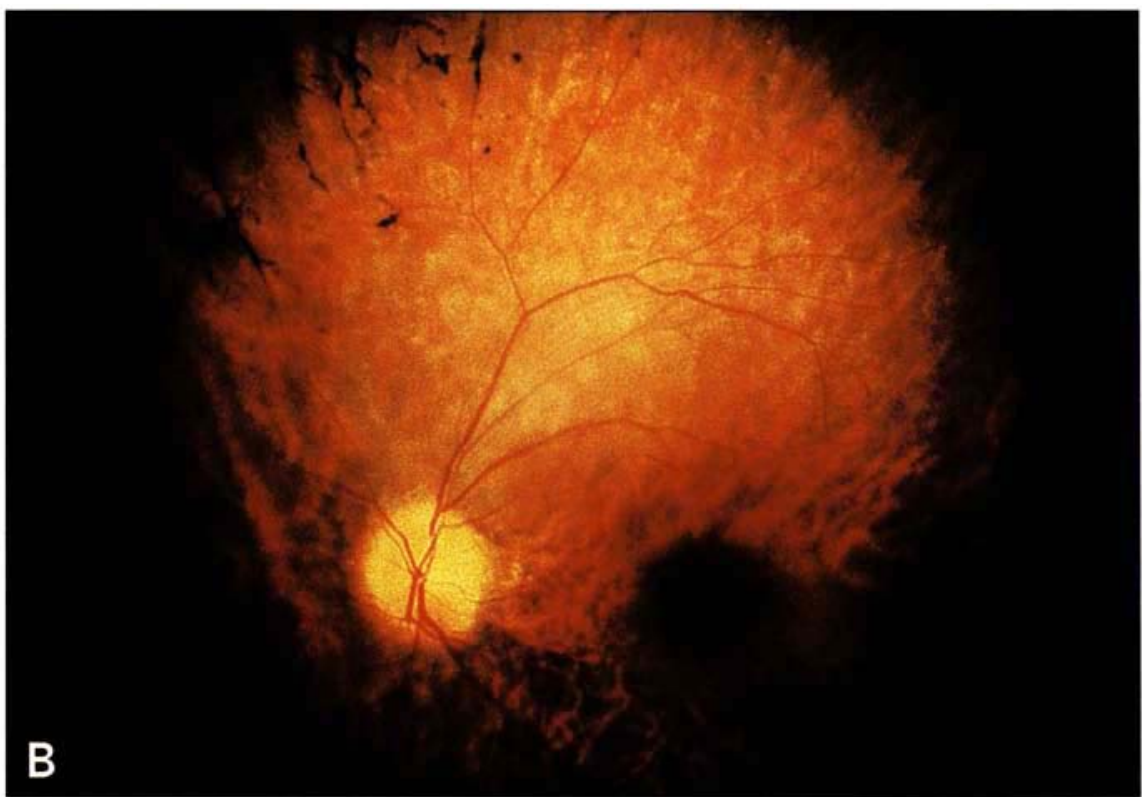

et permet d'exclure les autres causes de nystagmus du nouveau-né (causes neurologiques, hypoplasie papillaire, achromatopsie ou cécité nocturne congénitale essentiellement).

L'hétérogénéité génétique de cette affection a été suspectée depuis fort longtemps par l'observation d'unions entre sujets atteints ayant donné naissance à des enfants indemnes $(\mathrm{m} / \mathrm{s}$ $n^{\circ} 9$, vol. 5, p. 691), mais aucune étude de cartographie de cette maladie n'était publiée à ce jour. En 1991, nous avons d'abord sélectionné, grâce à la collaboration de plusieurs équipes, un échantillon de quinze familles à cas multiples. Ces quinze familles comportaient 38 sujets atteints et 55 apparentés indemnes. Six d'entre elles étaient consanguines, cinq venaient d'Afrique du Nord et dix étaient originaires de différentes régions de France. Nous avons tout d'abord opté pour une stratégie combinant étude de déséquilibre de liaison et approche "gène candidat ". Le bras long du chromosome 17 avait retenu notre attention en raison d'un lod-score indicatif d'une éventuelle liaison génétique avec une sonde biallélique au locus D17S40 (LEW101, $\mathrm{Zmax}=2,37$ à $\theta=0,05)$. Ce RFLP (restriction fragment length polymorphism) n'était pleinement informatif que pour 9 familles, huit étant liées au locus D17S40. Toutefois quatre microsatellites fortement informatifs flanquant le locus D17S40 permirent d'exclure cette région du bras long $m / s n^{\circ} 3$, vol. 11 , mars 95 17p. Enfin, des lod-scores négatifs furent obtenus pour le groupe des dix familles françaises (groupe 2) confirmant l'hétérogénéité génétique de notre échantillon. LCAI est le premier gène localisé dans cette redoutable maladie cécitante du nouveauné. Son identification apportera sans nul doute une meilleure compréhension de la physiopathologie du développement très précoce de la vision.

\section{Une maladie, plusieurs gènes..}

Le polymorphisme clinique est une notion ancienne, enseignée dans les premiers manuels de génétique [42] Depuis longtemps déjà, sont décrites l'irrégularité de la manifestation d'un gène, sa "pléiotropie " ou son étrange variabilité phénotypique, aboutissant parfois à un très large spectre de gravité pour une même maladie. Depuis longtemps aussi, est connue la possibilité de modes d'hérédité différents pour le même type d'affections, posant le problème délicat des formes sporadiques. Mais les progrès actuels de la génétique ont permis de mettre en lumière une diversité encore plus impressionnante et insoupçonnée jusqu'alors : c'est l'observation qu'une même maladie résulte de l'effet de plusieurs gènes indépendants situés en des points différents du génome mais dont les mutations peuvent induire des phénotypes très voisins. Cette hétérogénéité génétique contrastant avec une relative homogénéité clinique a été démontrée tout d'abord dans les maladies dominantes autosomiques. Citons pour exemple les maladies du collagène [43], les amyloidoses familiales [44], la maladie de Marfan et certaines affections du système nerveux périphérique [45]

La présente étude souligne le surprenant phénomène de l'hétérogénéité génétique dans une maladie récessive autosomique cliniquement homogène : la maladie de Usher. La localisation du premier gène responsable du syndrome de Usher type I sur le chromosome $14 \mathrm{q}$ dans une partie seulement des familles françaises souleva, en dépit de l'effet fondateur vraisemblable, quelques suspicions de la part de nos collègues d'outreAtlantique, malgré la signification des tests d'hétérogénéité. 


\section{RÉFÉRENCES}

45. Bird TD, Ott J, Giblett ER, Chance PF, Sumi S, Kraft GH. Genetic linkage evidence for heterogeneity in Charcot-Marie-Tooth neuropathy (HMSN type I). Ann Neurol $1983 ; 14: 679-84$.

46. Kwitek-Black A, Carmi R, Duyk GM, Buetow KH, Elbedour K, Parvari R, Yandava CN, Stone EM, Sheffield VC. Linkage of Bardet-Biedl syndrome to chromosome $16 \mathrm{q}$ and evidence for non-allelic genetic heterogeneity. Nature Genet 1993 ; 5: 392-6.

47. Leppert M, Baird L, Anderson KL, Otterud B, Lupski JR, Lewis RA. Bardet-Biedl syndrome is linked to DNA markers on chromosome $11 \mathrm{~g}$ and is genetically heterogeneous. Nature Genet 1994 ; 7 : 108-11.

48. Sheffield VC, Carmi R, Kwitek-Black A, Rokhlina T, Nishimura D, Duyk GM, Elbedour K, Sunden SL, Stone EM. Identification of a Bardet-Biedl syndrome locus on chromosome 3 and evaluation of an efficient approach to homozygosity mapping. Hum Mol Genet 1994 ; 3 : 1331-5.

49. Stone EM, Nichols BE, Kimura AE, Weingeist TA, Drack A, Sheffield VC. Clinical features of a Stargardt-like dominant progressive macular dystrophy with genetic linkage to chromosome 6q. Arch Ophthalmol $1994 ; 112: 765-72$.

50. Zhang K, Bither PP, Park R, Donoso LA, Seidman JG, Seidman CE. A dominant Stargardt's macular dystrophy locus maps to chromosome 13q34. Arch Ophthalmol 1994; 112 : $759-64$

51. Kajiwara K, Sandberg MA, Berson EL, Dryja TP. A null mutation in the human peripherin/rds gene in a family with autosomal dominant retinitis punctata albescens. Nature Genet 1993 ; 3 : 208-12.

52. Travis GH, Hepler JE. A medley of retinal dystrophies. Nature Genet $1993 ; 3$ : 191-

53. Rosenfeld PJ, Cowley GS, Mc Gee TL, Sandberg MA, Berson EL, Dryja TP. A null mutation in the rhodopsin gene causes rod photoreceptor dysfunction and autosomal recessive retinitis pigmentosa. Nature Genet
Il est vrai que le diagnostic de syndrome de Usher de type I est particulièrement aisé et que les signes observés chez les malades sont remarquablement similaires : surdité profonde dès la naissance, absence d'acquisition du langage, hypotonie constatée dès les premiers mois de vie à rapprocher du déficit vestibulaire bilatéral constant et, enfin, rétinopathie pigmentaire reconnue dès l'âge de 10 ans et décrite de la même façon avec les mêmes mots. Contrairement à ce que nous avions observé dans les rétinopathies pigmentaires liées au chromosome X [8], aucun détail, aucune note discordante ne séparaient un patient d'un autre. La localisation d'un gène sur le chromosome 14 dans une partie seulement des familles pouvait, il est vrai, surprendre, choquer, susciter le doute d'autant que Kimberling et al. et Smith et al. publiaient simultanément la localisation de deux autres gènes sur le chromosome 11, à grande distance l'un de l'autre (le dernier semblant également être le fruit d'un effet fondateur dans une population acadienne de Louisiane). Fort heureusement, nous avons pu montrer (1) qu'aucun locus ne rendait compte de l'ensemble des généalogies, (2) qu'en scindant notre échantillon en fonction de l'origine géographique des grands-parents, nous étions capables de confirmer la localisation d'un gène sur le chromosome $14 \mathrm{q}$ dans un isolat géographique du département des Deux-Sèvres et (3) que les familles restantes étaient compatibles avec une localisation, respectivement, sur les bras long et court du chromosome 11. Enfin, deux familles non françaises de notre échantillon, l'une turque et l'autre marocaine, ne correspondaient à aucun des trois loci, suggérant ainsi l'existence d'au moins quatre gènes à l'origine de cette affection au phénotype si régulier. Depuis les tribulations de la maladie de Usher, plusieurs exemples d'hétérogénéité génétique de dystrophies rétiniennes récessives autosomiques ont été rapportés, non pas tant les rétinopathies pigmentaires récessives autosomiques aux phénotypes variés, que le syndrome de Bardet-Biedl*, as-

* Syndrome malformatif associant une rétinite pig mentaire, une obésité, un retard intellectuel, une syndactylie ou une polydactylie et un hypogonadisme hypogonadotrophique. sociation malformative si particulière qui, lui aussi, correspond à l'altération d'au moins trois gènes différents dans des régions chromosomiques différentes (chromosomes 16q, 11q et $3 p$ [46-48]. La dernière localisation sur le chromosome 3 a été rendue possible, par la méthode de l'homozygotie par filiation, dans une grande famille consanguine de bédouins du désert du Neguev et il est intéressant de noter que c'était déjà dans une famille de bédouins du Neguev qu'avait été localisé le gène du chromosome $16 q$, par la même équipe qui souligne dans son étude qu'aucune discordance phénotypique ne sépare les deux pedigrees étudiés.

Ainsi, des maladies parfaitement homogènes cliniquement nous réservent-elles des surprises génotypiques considérables, démontrant la complexité des rapports entre la présentation clinique et la structure ou la fonction du matériel génétique.

\section{Un gène, plusieurs maladies}

Le chemin vers la connaissance passe par l'apprentissage de notions nouvelles, controversées aussitôt qu'elles sont énoncées. La recherche dans le domaine des dystrophies rétiniennes en fournit une preuve supplémentaire. Ainsi, la maladie de Stargardt dans sa forme typique, précoce, et le fundus favimaculatus, plus tardif, manifestent une grande diversité clinique d'une famille à l'autre, bien que l'atteinte rétinienne y soit cantonnée aux régions maculaire et para-maculaire. La lésion primaire n'étant pas connue, un vaste champ s'ouvrait naturellement aux spéculations sur l'hétérogénéité ! Le Pr. Alan Bird qui recevait l'Action Concertée des Communautés Européennes, en mars 1993, au Moorfield Eye Hospital de Londres déclarait qu'à son avis «la maladie de Stargardt n'existait pas " et que cette terminologie regroupait très probablement plusieurs maculopathies d'origine génétique différente... C'est dans ce contexte peu enthousiasmant que nous avons débuté nos travaux de cartographie de la maladie de Stargardt. Ainsi, nous étionsnous entourés des meilleures garanties d'homogénéité clinique et donné les meilleures chances de re- 
pérer un gène responsable, même en situation d'hétérogénéité génétique. C'est pourquoi nous fûmes les premiers surpris lorsque nous mîmes en évidence une liaison forte et homogène sur l'ensemble de l'échantillon avec un marqueur polymorphe du bras court du chromosome 1 , en dépit des origines très différentes des familles (diverses régions de France, Italie, Maroc) (m/s $n^{\circ} 12$, vol. 9, $p$. 1418). Compte tenu de la petite taille de cet échantillon, la prudence empêchait d'exclure la possibilité d'un autre locus. Aussi, avons-nous voulu tester les formes tardives de fundus flavimaculatus, ces maladies d'évolution plus lente et de meilleur pronostic. Nous sommes parvenus à la conclusion qu'en dépit de leurs différences cliniques (début de 17 à 60 ans, évolution très lente et acuité visuelle préservée pendant quinze à vingt ans d'évolution), les deux phénotypes distincts correspondaient, en fait, au même locus chromosomique. Enfin, très récemment, Anderson et al. rapportèrent l'étude de trentequatre familles nord-américaines et de deux grandes familles consanguines d'Arabie Saoudite, atteintes de la maladie de Stargardt et confimèrent l'homogénéité génétique de cette affection au locus 1p13 (American Society for Human Genetics, Montréal, octobre 1994).

Pour rendre compte de ces observations, on est en droit d'émettre plusieurs hypothèses :

(1) Il n'existe qu'un seul gène responsable de ces dystrophies maculaires, les distinctions cliniques s'expliquant par des mutations différentes.

(2) Il existe sur le chromosome $1 \mathrm{p} 13$ une batterie de gènes codant pour l'intégrité de l'épithélium pigmentaire maculaire.

(3) Il existe un continuum de dystrophies maculaires à ce locus, de la toute première enfance aux âges avancés de la vie, ouvrant le débat sur l'interaction possible d'un gène majeur avec des gènes modificateurs, en particulier dans les très fréquentes et invalidantes dégénérescences maculaires liées à l'âge.

A une époque où l'hétérogénéité génétique constitue l'obstacle majeur à la poursuite de la cartographie primaire des maladies héréditaires, il est surprenant de constater une telle ho- mogénéité, et cela d'autant que certaines maculopathies dominantes autosomiques viennent d'être localisées dans deux régions chromosomiques différentes (chromosome $6 \mathrm{q}$ et $13 \mathrm{q}$, $[49,50]$.

\section{L'hétérogénéité moléculaire}

L'analyse moléculaire des gènes déjà identifiés pour les rétinopathies pigmentaires apporte des exemples sans cesse plus nombreux de l'extraordinaire diversité des mutations à chaque locus. Soixante-dix mutations dans le gène de la rhodopsine ont été publiées à ce jour pour les rétinopathies pigmentaires dominantes autosomiques sans que l'on puisse faire correspondre une forme clinique à une mutation [27]. Nous avons décrit une mutation de rétinopathie pigmentaire dans une forme "albescente" de la maladie [28], ce phénotype particulier rapporté au gène de la périphérine [51]. De fait, les mutations de la périphérine peuvent donner lieu à une rétinite pigmentaire typique $\left(m / s n^{\circ} 2\right.$, vol. $\left.8, p .171\right)$ ou à diverses formes de dystrophie maculaire $\left(m / s n^{\circ} 4\right.$, vol. 9, p. 478) [52].

Poursuivant sur le chemin de la complexité, le fait que des modes de transmission différents pouvaient exister pour un même locus fut démontré pour la rhodopsine, incriminée à la fois dans des formes dominantes et des formes récessives autosomiques de rétinopathie pigmentaire $\left(m / s n^{\circ} 1\right.$, vol. $8, p$. 82) [53]. Enfin, la très récente mise en évidence d'un digénisme à l'origine de rétinite pigmentaire s'installant à la manière d'un caractère récessif autosomique, se transmettant ensuite comme un caractère dominant, mais à ségrégation de 1/4 (au lieu de 1/2), nous apparaît comme étant la plus surprenante des notions nouvelles. Ainsi, deux protéines (périphérine et protéine ROM1) codées par des gènes à localisation chromosomique différente (6p et $11 q$ ) s'associent-elles pour induire une maladie chez les doubles hétérozygotes, notion qui aurait été jugée extravagante il y a encore cinq ans! $\left(m / s n^{\circ} 8-9\right.$, vol. 10, p. 908) [51]. Si exceptionnel que nous semble aujourd'hui ce cas de figure, il pourrait bien ne pas être unique ! Cela pourrait, d'une part, accroître d'autant la complexité des modes de transmission possibles dans les rétinopathies pigmentaires, mais, d'autre part et surtout, remettre en cause la solidité du conseil génétique devant une transmission récessive apparemment non ambiguē de la maladie

\section{Summary}

Genes for childhood retinal dystrophies

The group of retinal dystrophies represent a frequent cause of blindness in childhood (200000 affected individuals in Europe). Its clinical, genetic and molecular heterogeneity has been recently emphasized. This group includes three clinically recognizable conditions, namely Usher syndrome type I, Stargardt's disease and Leber's congenital amaurosis. We have mapped a gene for Usher syndrome type I to chromosome $14 q 32$ in fifteen families of western France ancestry (Poitou). The gene for Stargardt's disease has been mapped to chromosome 1p21-p13. This disease is genetically homogeneous and we have recently shown that Stargardt's disease and late-onset fundus flavimaculatus with macular dystrophy are most probably allelic conditions. Finally, a gene for Leber's congenital amaurosis has been mapped to chromosome $17 p$ by homozygosity mapping in five consanguineous families of maghrebian ancestry, but this condition proved to be genetically heterogeneous in our series.

\section{Remerciements}

Nous remercions chaleureusement Gisèle Gal pour sa collaboration. Ces travaux n'auraier pas été possibles sans le soutien de l'Association Française contre les Myopathies (AFM) l'Association Française Retinitis Pigmentos (AFRP) et l'Institut Électricité Santé.

\section{TIRÉS À PART}

A. Munnich. 